\title{
Superthermal ion signatures of auroral acceleration processes
}

Article

Published Version

Moore, T. E., Chappell, C. R., Lockwood, M. and Waite, J. H. (1985) Superthermal ion signatures of auroral acceleration processes. Journal of Geophysical Research, 90 (A2). pp. 1611-1618. ISSN 0148-0227 doi: https://doi.org/10.1029/JA090iA02p01611 Available at https://centaur.reading.ac.uk/38917/

It is advisable to refer to the publisher's version if you intend to cite from the work. See Guidance on citing.

Published version at: http://dx.doi.org/10.1029/JA090iA02p01611

To link to this article DOI: http://dx.doi.org/10.1029/JA090iA02p01611

Publisher: American Geophysical Union

All outputs in CentAUR are protected by Intellectual Property Rights law, including copyright law. Copyright and IPR is retained by the creators or other copyright holders. Terms and conditions for use of this material are defined in the End User Agreement.

\section{www.reading.ac.uk/centaur}

\section{CentAUR}

Central Archive at the University of Reading 
Reading's research outputs online 


\title{
Superthermal Ion Signatures of Auroral Acceleration Processes
}

\author{
T. E. MOORE, C. R. CHAPPELL, M. LOCKWOOD, AND J. H. WAITE, JR.
}

Space Science Laboratory, NASA Marshall Space Flight Center, Huntsville, Alabama

\begin{abstract}
The retarding ion mass spectrometer on the Dynamics Explorer 1 spacecraft has generated a unique data set which documents, among other things, the occurrence of non-Maxwellian superthermal features in the auroral topside ionosphere distribution functions. In this paper, we provide a representative sampling of the observed features and their spatial morphology as observed at altitudes in the range from a few thousand kilometers to a few earth radii. At lower altitudes, these features appear at auroral latitudes separating regions of polar cap and subauroral light ion polar wind. The most common signature is the appearance of an upgoing energetic tail having conical lobes representing significant ion heat and number flux in all species, including $\mathrm{O}^{+}$. Transverse ion heating below the observation point at several thousand kilometers is clearly associated with $\mathrm{O}^{+}$outflows. In some events observed, transverse acceleration apparently involves nearly the entire thermal plasma, the distribution function becomes highly anisotropic with $T_{\perp}>T_{\| \mid}$, and may actually develop a minimum at zero velocity, i.e., become a torus having as its axis the local magnetic field direction. At higher altitudes, the localized dayside source region appears as a field aligned flow which is dispersed tailward across the polar cap according to parallel velocity by antisunward convective flow, so that upflowing low energy $\mathrm{O}^{+}$ions appear well within the polar cap region. While this flow can appear beamlike in a given location, the energy dispersion observed implies a very broad energy distribution at the source, extending from a few tenths of an $\mathrm{eV}$ to in excess of $50 \mathrm{eV}$. On the nightside, upgoing ion beams are found to be latitudinally bounded by regions of ion conics whose half angles increase with increasing separation from the beam region, indicating low altitude transverse acceleration in immediate proximity to, and below, the parallel acceleration region. These observations reveal a clear distinction between classical polar wind ion outflow and $\mathrm{O}^{+}$enhanced superthermal flows, and confirm the importance of low altitude transverse acceleration in ionospheric plasma transport, as suggested by previous observations.
\end{abstract}

\section{INTRODUCTION}

The evidence that the terrestrial ionosphere is at times the source of much of the $\mathrm{keV}$ plasma within the earth's magnetosphere [Johnson, 1979; Horwitz, 1982] has brought an increasing awareness that planetary ionospheres are active participants in energetic magnetospheric processes. Processes associated with discrete auroral arcs result in the production of upflowing ion beam and conic distributions having characteristic energies in the $\mathrm{keV}$ range [Chiu et al., 1983], yielding a source of energetic ionospheric plasma operating in the range of altitudes above about 1 earth radius. Ionospheric observations have also revealed that the magnetosphere is at times a notable sink of ionospheric plasma, producing significant departures from diffusive equilibrium [Lockwood and Titheridge, 1982]. It has been suggested as well that an important component of this ionosphere-magnetosphere transport is the superthermal heating or acceleration of ionospheric ions at low altitudes in the auroral topside $F$ region [Moore, 1984, and references therein].

Recently acquired observations, obtained from the retarding ion mass spectrometer (RIMS) aboard the Dynamics Explorer 1 (DE 1) spacecraft, include the first mass discriminating measurements of topside thermal plasma to reveal both the thermal flows (polar wind) and superthermal effects due to auroral processes. The purpose of this paper is to provide a representative event sampling of the range of superthermal effects observed by RIMS at altitudes from a few thousand $\mathrm{km}$ to a few earth radii.

This paper is not subject to U.S. copyright. Published in 1985 by the American Geophysical Union.

Paper number 4A8250.
A related study of large fluxes of upflowing superthermal $\mathrm{O}^{+}$ions observed by DE 1 RIMS in the polar cap during disturbed conditions has been reported by Waite et al. [1985]. A complementary study of the statistical occurrence morphology for the low altitude events discussed here is the subject of M. Lockwood et al. (unpublished manuscript, 1984).

Following this opening discussion. we provide a brief summary of the salient charactertistics of the RIMS instrument. We then outline the basis for selection of events for this study. Four events are documented and analyzed in some detail. A general discussion relating and comparing the events is given next, followed by a statement of conclusions suggested by these observations.

\section{INSTRUMENTATION}

The retarding ion mass spectrometer consists of three nearly identical sensor heads, one viewing radially outward perpendicular to the DE-1 spacecraft spin axis, and two viewing in opposite directions aligned with the spin axis. The sensor heads are referred to as radial $(R),+Z,-Z$, respectively. Each head consists of a conventional retarding potential analyzer (RPA) assembly containing a grid which is biased in the range $0-50 \mathrm{~V}$ with respect to the spacecraft frame, and an electrometer collector containing a slit which passes ions to a conventional magnetic sector mass spectrometer having two mass channels (low and high) sampled by high rate channeltrons. All three head assemblies, including large entrance aperture planes, may be biased 2,4 or $8 \mathrm{~V}$ negative relative to the spacecraft frame in order to overcome positive spacecraft floating potentials relative to the plasma. However, all of the data examined here were taken in a mode for which this bias was set to zero.

The typical mode of operation involved the sampling of 
four to eight ion species at 15 or 30 exponentially spaced retarding potentials with a basic sampling interval of 0.012 s. With a spin period of $6 \mathrm{~s}$, adequate RPA-spin data arrays for each ion species are obtained in several spin periods, i.e., about $30 \mathrm{~s}$. Angular features larger than approximately $15^{\circ}$ are resolved by the radial head.

The RIMS count rate is proportional to the integral flux above that energy corresponding to the sum of the spacecraft potential and the retarding potential, i.e., the first velocity moment of the distribution function beyond that energy and within the angular field of view. One qualification of this statement is that each mass channel responds only to ions within an energy range with a width of about $250 \mathrm{eV}$ for $\mathrm{H}^{+}, 75 \mathrm{eV}$ for $\mathrm{He}^{+}$, and $15 \mathrm{eV}$ for $\mathrm{O}^{+}$. In general, the energy pass band is swept upward with the retarding potential so that ions just clearing the potential barrier are always at energy band center. As a consequence, the retarding potential sweep to $50 \mathrm{~V}$ provides a differential response to $\mathrm{O}^{+}$distributions extending to energies larger than $10-20 \mathrm{eV}$. In addition, the integral flux is weighted by an energy dependent solid angle aperture, though this effect is appreciable only for superthermal tails having characteristic energies of tens of $\mathrm{eV}$ and higher.

RIMS is self-calibrating in flight through comparison of the electrometer and mass spectrometer response. Variations in channeltron gain due to high count rate fatigue may be monitored and compensated. Early in the DE-1 mission, prior to any channeltron detector degradation, the effective geometry factors for rammed thermal ions are as follows: $\mathrm{H}^{+}$(low-mass channel) $2.7 \times 10^{-3} \mathrm{~cm}^{2} \mathrm{sr}, \mathrm{He}^{+}$ (low-mass channel) $2.7 \times 10^{-3} \mathrm{~cm}^{2} \mathrm{sr}, \mathrm{He}^{+}$(high-mass channel) $4.3 \times 10^{-3} \mathrm{~cm}^{2} \mathrm{sr}$, and $\mathrm{O}^{+}$(high-mass channel) $1.9 \times 10^{-3} \mathrm{~cm}^{2} \mathrm{sr}$. Consequently, except where detector degradation is noted, the corresponding ratios of integral flux $\left(\mathrm{cm}^{-2} \mathrm{~s}^{-1} \mathrm{sr}^{-1}\right)$ to count rate (per sample) are $3.1 \times$ $10^{4}, 3.1 \times 10^{4}, 1.9 \times 10^{4}$, and $4.4 \times 10^{4}$, respectively.

It is possible to differentiate (or deconvolve, in the case of $\mathrm{O}^{+}$) the RIMS RPA curves and deduce the distribution function, albeit with some increase of uncertainty due to the differencing procedure. The RIMS radial head suffered a failure of its RPA bias supply on day 329 of 1981. However, much useful information is still obtained from the spin distributions, which are for zero RPA bias essentially first velocity moments of the entire distribution above spacecraft potential. Further details concerning RIMS may be found in the work by Chappell et al. [1982].

\section{OBSERVATIONS}

Four events will be discussed in this section. They have been selected as representative of the phenomena observed in the nightside and dayside auroral zone at "high" and "low" altitudes. The choice of this organization is heavily influenced by the early orbit of DE 1 (elliptical, $3.6 R_{E}$ by $700 \mathrm{~km}$ in altitude, $90^{\circ}$ nominal inclination, $1000-2200$ local time initially) and by the influence of altitude (or density) on the spacecraft potential. The prevalence of the low altitude events presented below is discussed by $\mathbf{M}$. Lockwood et al. (unpublished manuscript, 1984], who present a statistical study of the occurrence morphology of $\mathrm{O}^{+}$events based upon the full DE-RIMS data set acquired between October 1981 and October 1983.

Events are categorized as high altitude when the low energy core of the $\mathrm{H}^{+}$distribution is not visible above the positive spacecraft potential. This indicates positive potential greater than approximately one volt, and total ion density less than about $100 \mathrm{~cm}^{-3}$. At times, a cold rammed $\mathrm{O}^{+}$core will be observed at high altitudes, due to the larger ram energy of $\mathrm{O}^{+}$(few eV for typical ram speeds). Events fall into the low altitude category when densities are large enough and spacecraft potential low enough for low energy cores of the light ion distributions to be visible. A common feature of such periods is a clear shift of the light ion spin distributions away from the spacecraft ram direction, indicating upward field aligned flow. The cold rammed $\mathrm{O}^{+}$present at these times generally shows small shifts (less than the thermal width of the spin angle distribution) from the ram direction. This feature thus represents polar wind light ion flow relative to the $\mathrm{O}^{+}$and is, as expected, a common feature of low altitude data periods when DE 1 is outside the plasmasphere. During high altitude passes over the polar regions, the light ion flows tend to be obscured by positive spacecraft potential, but can be observed using the RIMS aperture bias mode [Nagai et al., 1984]. The low altitude auroral zone events studied here appear embedded between polar cap and subauroral regions of light ion polar wind.

\section{High Altitude Nightside (Day 293, 1983)}

Plate 1 illustrates a common nightside auroral zone signature using RPA potential and spin phase spectrograms for (Plate $1 a) \mathrm{H}^{+}$and (Plate $\left.1 b\right) \mathrm{O}^{+}$. (Plate 1 can be found in the separate color section in this issue.) $\mathrm{He}^{+}$is not shown but its behavior is qualitatively similar to that of the $\mathrm{H}^{+}$. The contouring indicates spin averaged integral flux for energies above the retarding potential given on the logarithmic scale at the right in the upper panel for each species, while the lower panels provide the RPA sweep averaged integral flux within spin angle bins indicated at the right in relation to the spacecraft ram direction. The white traces in the spin-time spectrograms indicate the spin phase of closest approach to parallel and antiparallel to the local magnetic field, the upper of the two indicating the upgoing field aligned direction.

The spacecraft is traveling equatorward from the north polar cap into the plasma sheet at evening local times and at altitudes near $1.75 R_{E}$. Note the lack of any cold rammed $\mathrm{H}^{+}$flux in the ram direction, consistent with low ion densities and significant positive spacecraft potential. Some $\mathrm{O}^{+}$is observed in the ram direction before and after the event at 0010 to 0020 UT. This event exhibits an $\mathrm{X}$-shaped signature in the spin phase spectrogram format, indicating a conical pitch angle distribution at the high latitude edge of the event, which is skewed toward the ram direction at $\mathrm{O}^{+}$; evloving to a field aligned flow, then returning to a conical pattern at the equatorward edge of the event. In $\mathrm{O}^{+}$, the conical character is by no means as apparent as that of the $\mathrm{H}^{+}$in this presentation. However, it is clear that observable fluxes of $\mathrm{O}^{+}$are present at energies up to and exceeding $50 \mathrm{eV}$ in the field-aligned center of the event. This is also true of the $\mathrm{H}^{+}$and $\mathrm{He}^{+}$at that time.

In order to more clearly show the characteristics of this event, Figures $1 a-1 f$ provide contour plots of the full RPA-spin data array for $\mathrm{O}^{+}$during six subintervals of this 


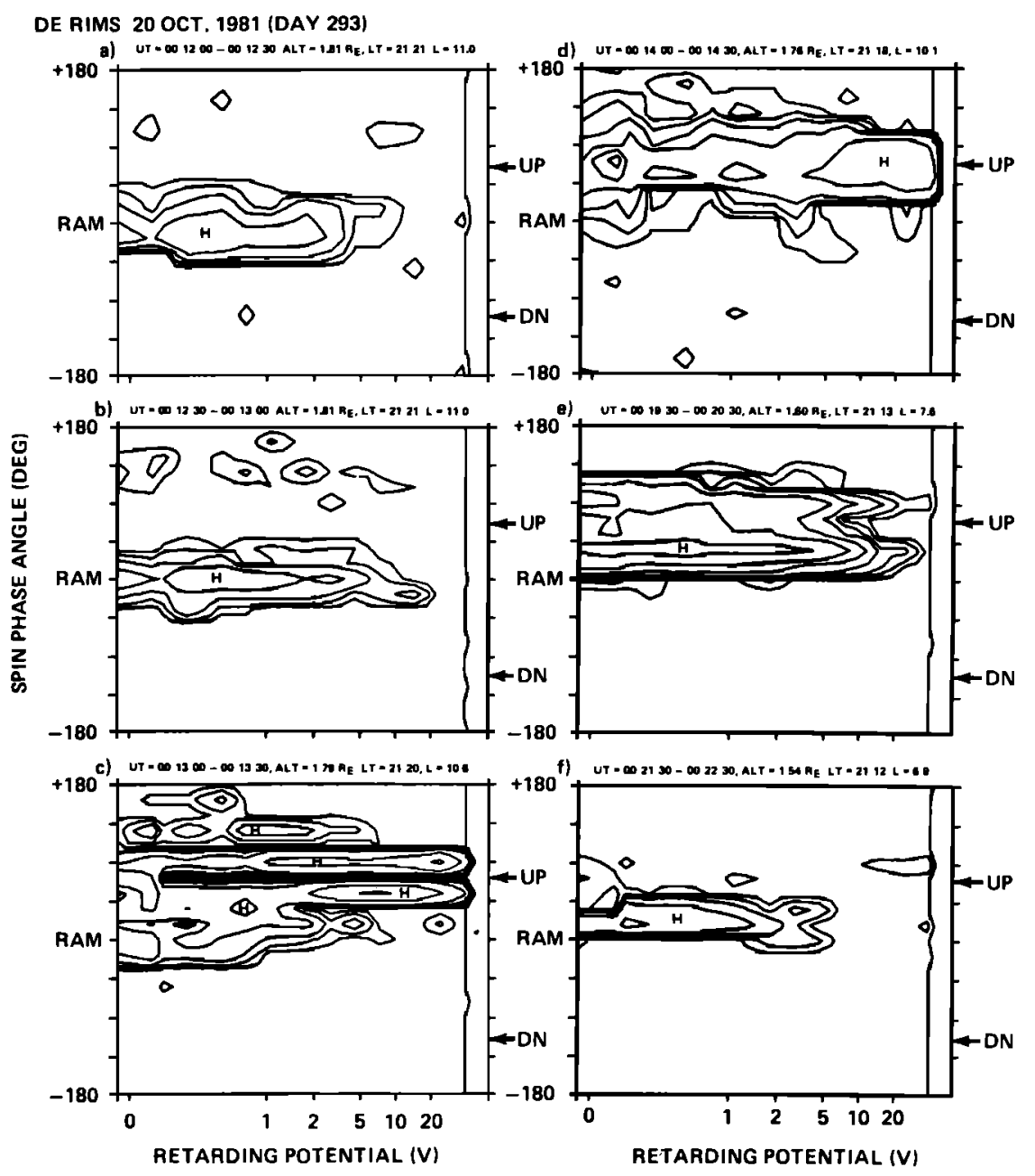

Fig. 1. DE 1 RIMS retarding potential-spin data matrix contour plots for subintervals of the data period shown in Plate 1. Contours appear at intervals of a factor of 2 , local maxima are indicated by the symbol $\mathrm{H}$, and arrows at the right of each frame indicate the upward and downward field-aligned directions.

period. These plots provide considerable information not apprent in the spectrograms, which have been averaged over one or the other of the array dimensions. Note that the magnetic field direction in these plots is indicated by the arrows marking minimum (upgoing) and maximum (downgoing) pitch angle. In these plots, the integral flux for a given RPA step is represented by contours separated by a factor of two. Local maxima are indicated with the symbol $\mathrm{H}$.

Figure $1 a$ shows the $\mathrm{O}^{+}$distribution at the time when the $\mathrm{H}^{+}$and $\mathrm{He}^{+}$conics first appear. Note that the $\mathrm{O}^{+}$is a cold rammed population which is retarded at potentials of a few volts, consistent with the ram energy of $\mathrm{O}^{+}$for a spacecraft speed of $6.5 \mathrm{~km} / \mathrm{s}$. As the $\mathrm{H}^{+}$conic "folds" toward the magnetic field direction (see Figure 1, 0013 $\mathrm{UT}$ ), Figure $1 b$ shows the beginnings of a conic appearing in $\mathrm{O}^{+}$. The next $30 \mathrm{~s}$ interval (Figure $1 c$ ) shows that a very pronounced $\mathrm{O}^{+}$conic has formed, which is just resolved by RIMS with a cone half angle of $15^{\circ}-20^{\circ}$. At this time, the $\mathrm{H}^{+}$conic has folded sufficiently that it is no longer resolved in the RIMS radial detector. The $\mathrm{O}^{+}$participation in the conical flow is clearly enhanced as the conic half angle is reduced. The conic half angle may be taken as indicative of the altitude of acceleration, assuming insignificant field aligned potential drop. The continued presence of cold rammed $\mathrm{O}^{+}$during the subinterval in Figure $1 c$ suggests that the parallel potential drop below the spacecraft is very small if not zero. It may then be inferred that the enhanced $\mathrm{O}^{+}$flux is associated with transverse acceleration in the range of $1000-2000 \mathrm{~km}$ altitude in this case, the range reflecting the uncertainty in conic half angle.

Figure $1 d$ shows that an $\mathrm{O}^{+}$beam formed in the center of the event (recall that the $\mathrm{O}^{+}$channel responds differentially with an energy bandwidth of approximately 15 $\mathrm{eV})$. Figures $1 e$ and $1 f$ show that $\mathrm{O}^{+}$conics were again seen in the low latitude part of the structure, this time with wider conic half angles, prior to a return to cold rammed $\mathrm{O}^{+}$. This suggests that part (of order $50 \mathrm{~V}$ ) of the auroral parallel electric field extended below the spacecraft at the center of this structure, and that conic generation was associated with the edges of the electric field structure. Ion data from the DE 1 high altitude plasma instrument show that the ion "beam" actually still contained a conical structure (J. D. Winningham, private communication, 1984), extending beyond the RIMS energy range.

\section{Low Altitude Nightside (Day 110, 1982)}

Plate 2 illustrates the clear appearance of transverse acceleration at somewhat lower altitude on the nightside. (Plate 2 can be found in the separate color section in this 

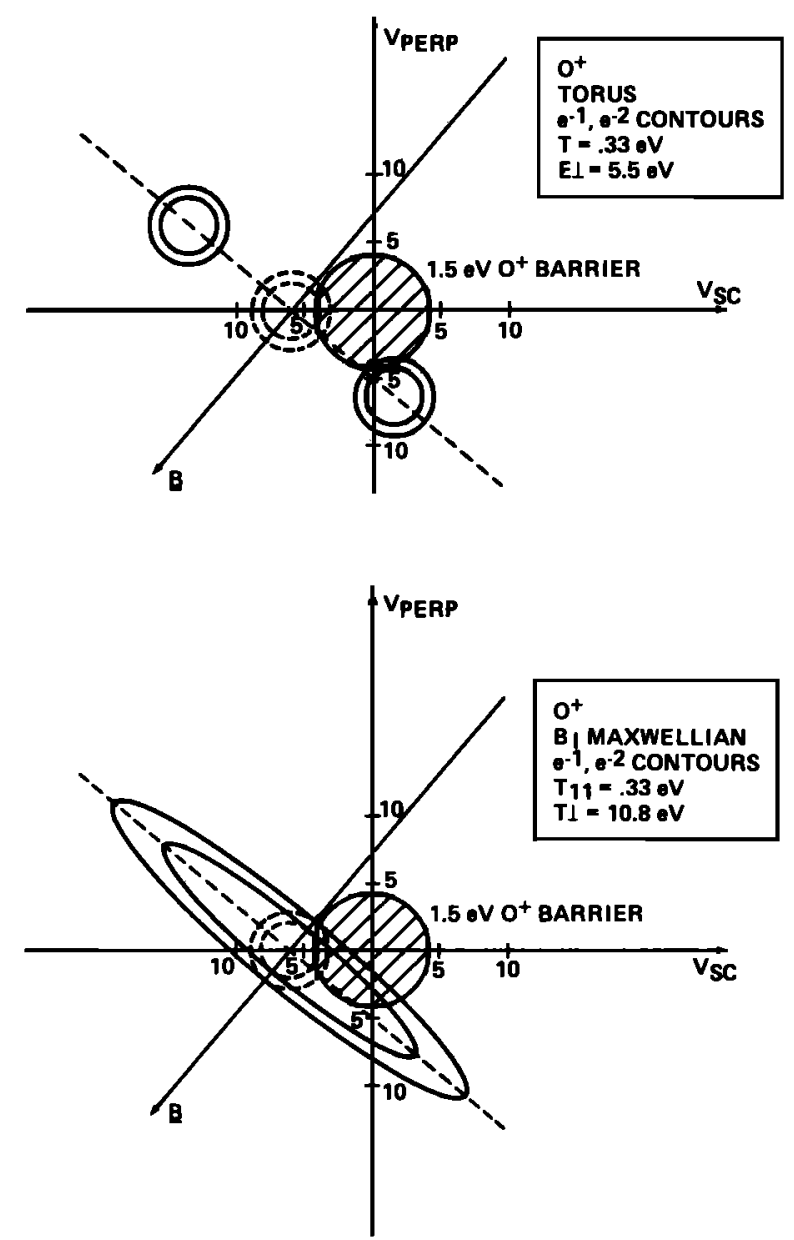

Fig. 2. Schematic illustration of interpretations of the data of Plate 2. Axes represent velocity space in the frame of the moving spacecraft, $V_{\text {sc. }}$ The magnetic field lies nearly in this plane and is indicated as an appropriately oriented line. The upper panel shows a toroidal distribution function while the lower panel shows a biMaxwellian. Cross-hatched circles indicate the region obscured by a $+1.5 \mathrm{~V}$ spacecraft potential. Dashed circles indicate typical isotropic Maxwellian distributions.

issue.) The format of Plate 2 provides spin phase spectrograms for $\mathrm{O}^{+}, \mathrm{He}^{+}$, and $\mathrm{H}^{+}$. The spacecraft motion is low across the southern polar cap, rising into the evening plasma sheet. There is significant outflow of $\mathrm{H}^{+}$in the polar cap, and some downward or convective flow of $\mathrm{He}^{+}$and $\mathrm{O}^{+}$. The plasma is basically cold rammed until $0844 \mathrm{UT}$, when, without a large discontinuity in flux magnitudes, there is an extremely abrupt change in the angular distributions. What had been a single angular peak near the ram direction becomes two separate peaks with minima near the magnetic field aligned directions. This immediately suggests transverse acceleration. Moreover, the subsequent evolution of the angular distribution peaks of each species toward the upgoing magnetic field direction suggests that the spacecraft is flying over a transverse acceleration region relative to which it is ascending. However, note that the rate of "folding" of these evolving conical distributions decreases with increasing mass per unit charge. This suggests that the simple picture of parallel acceleration by the magnetic mirror force is complicated for these ions by gravitational effects. Another noteworthy feature of this event is its broad extent, beginning at an $L$ value of 13 and extending down to $L=7.3$, where data collection for this pass ended.

The type of transverse acceleration apparent in this event is extreme in that essentially all of the thermal ions participate in the transverse acceleration, leaving no cold rammed plasma at all. The signature expected for local transverse heating yielding $T_{\perp}>T \|$, i.e., a bi-Maxwellian plasma, is a broadening of the spin distribution toward both directions perpendicular to the magnetic field, while maintaining a single peak at zero velocity (in the antiram direction in the spacecraft frame). The observed splitting of the cold rammed distributions into two distinct lobes could be produced in one of two ways: (1) transverse acceleration which is so effective that few zero speed ions remain, i.e., the distribution becomes toroidal; or (2) transverse heating to a bi-Maxwellian form in the presence of a positive spacecraft potential excluding ions with small velocity relative to the spacecraft. It should be noted that spacecraft potentials sufficient to exclude parts of the $\mathrm{O}^{+}$ distribution will also exclude larger fractions of the $\mathrm{He}^{+}$ and $\mathrm{H}^{+}$distributions. Therefore, on the basis of the presence of rammed $\mathrm{H}^{+}$and $\mathrm{He}^{+}$just prior to 0845 , we can set an upper limit on the positive spacecraft potential of $0.5 \mathrm{~V} \mathrm{H}^{+}$ram energy plus a few times the $\mathrm{H}^{+}$thermal energy. For reasonable thermal energies an upper limit of approximately $1.5 \mathrm{~V}$ is appropriate. The lack of a discontinuity of ion flux at 0845 precludes a discontinuity in the spacecraft potential, so that this same upper limit should apply just after 0845 , when the spin distribution split into two lobes.

Figure 2 schematically illustrates the way in which toroidal or bi-Maxwellian distributions could lead to the angular flux distributions of Plate 2. Both panels exhibit velocity space in the spin plane frame of reference of the DE 1 spacecraft. The axes represent the direction of motion of the spacecraft $\left(V_{S c}\right)$ and the perpendicular upward direction lying in the spin plane. The direction of the magnetic field at 0845 is indicated by the line through the location of cold rammed plasma in this reference frame. In the upper panel the contours represent a toroidal distribution function for $\mathrm{O}^{+}$having a thermal speed of $2 \mathrm{~km} / \mathrm{s}$ $(0.33 \mathrm{eV})$, and a torus radius of $8 \mathrm{~km} / \mathrm{s}(5.5 \mathrm{eV})$. In the lower panel, the contours represent an $\mathrm{O}^{+}$bi-Maxwellian having $T_{\perp}=10.8 \mathrm{eV}, T_{\|}=0.33 \mathrm{eV}$ and no field aligned or convection drift. The circles centered at the origin represent the region of velocity space not observable on account of positive $1.5 \mathrm{~V}$ potential for the $\mathrm{O}^{+}$ions. Note that the corresponding light ion velocity distributions would be broader for a given temperature than for $\mathrm{O}^{+}$ as shown. Recall that RIMS responds to the weighted first velocity moment of the distribution function along its (rotating) line of sight in velocity space, excluding those portions within a circle corresponding to the positive spacecraft potential.

The lower panel of the figure shows that positive potentials of $1.5 \mathrm{~V}$ or larger magnitude may be capable of producing the observed type of flux minimum in the field aligned direction when viewing a bi-Maxwellian distribution function. However, this is an extreme upper limit; the actual potential is probably considerably lower. Moreover, even with this large positive potential, the integral flux minimum parallel to the magnetic field should not exceed a factor of 2-3. Therefore, a distribution function having a minimum at zero speed in the plasma frame, 
i.e., toroidal, would appear to be a likely possibility. Whether the distribution is toroidal or bi-Maxwellian, it is clear that transverse heating in this event must affect the bulk of the ion distribution and that perpendicular temperatures of order $10 \mathrm{eV}$ are produced.

\section{High Altitude Dayside (Day 295, 1981)}

Plate 3 illustrates the type of signature seen at high altitude on the dayside, using RPA and spin-time spectrograms for $\mathrm{H}^{+}, \mathrm{He}^{+}$, and $\mathrm{O}^{+}$, with the spin spectrograms at the left and the RPA spectrograms at the right. (Plate 3 can be found in the separate color section in this issue.) Note that in this pass, the spacecraft stays close to 1000 local time, passing poleward over the dayside auroral zone. The top left panel shows conical and field aligned angular distributions in $\mathrm{H}^{+}$, separated from each other by the magnetospheric cusp or cleft as marked by diffuse unretarded ions (0945 UT). Although RIMS was not designed to observe energetic ions, and does not properly resolve them, the large fluxes of the cusp region produce this easily recognizable signature whose association with the cusp has been verified by comparison with the high altitude plasma instrument (HAPI) (J. Burch, private communication, 1984).

The $\mathrm{H}^{+}$conic and field aligned flow structures are qualitatively similar to the nightside $\mathrm{H}^{+}$features, though differing in spatial morphology. Close comparison with the $\mathrm{He}^{+}$and $\mathrm{O}^{+}$reveals a very interesting pattern during this and other high altitude dayside passes. Note that an $\mathrm{He}^{+}$field-aligned flow appears somewhat poleward of the $\mathrm{H}^{+}$field-aligned outflow (after $0953 \mathrm{UT}$ ), while an $\mathrm{O}^{+}$ flow appears yet further poleward than $\mathrm{He}^{+}$(mostly after 1003 UT). Further, as may be seen from the RPA spectrograms on the right, each species exhibits clear energy dispersion, less energetic ions of a given species appearing further poleward. It seems clear that the outflowing ions originate in a latitudinally localized source and are dispersed over the polar cap by the same geomagnetic velocity filter which produces similar dispersion of the magnetosheath ions entering the cusp region [Burch et al., 1982; Reiff et al., 1977]. The continuous nature of the energy dispersion observed here indicates that the field aligned outflows contain a broad spectrum of parallel energies, and are not beams in the sense of having a well defined peak in energy, unlike the nightside auroral zone structures. A second acceleration region appears at about 1013 $\mathrm{UT}$, which appears mainly to accelerate $\mathrm{O}^{+}$which originated in the more equatorward source region and has been separated from the $\mathrm{H}^{+}$and $\mathrm{He}^{+}$by the velocity filter effect.

Waite et al. [1985] have examined numerous DE polar cap passes and shown that very low-energy supersonic $\mathrm{O}^{+}$ outflows are found throughout the polar cap in association with strong magnetic activity and hence strong polar cap convection. They argued that the points of $\mathrm{O}^{+}$observation are consistent with convection from a source region in the dayside auroral oval. The present observations support this conclusion, providing a clear example of the spatial morphology which arises from the presence of a localized source of ions with comparable energy distributions, combined with a velocity filter consisting of crossed magnetic and electric fields: a geophysical mass spectrome ter. During times of very strong convection, such velocity filtered flows of $\mathrm{O}^{+}$should be a strong source of very low energy $\mathrm{O}^{+}$ions for the nightside auroral acceleration regions (M. Lockwood et al., unpublished manuscript, 1984).

\section{Low Altitude Dayside (Day 114, 1982)}

Plate 4 illustrates a very common signature of the dayside auroral ionosphere which is believed to be the low altitude source for the high altitude outflows described in the previous section (M. Lockwood et al., unpublished manuscript, 1984). (Plate 4 can be found in the separate color section in this issue.) The format of Plate 4 provides spin spectrograms for $\mathrm{O}^{+}$and $\mathrm{He}^{+}$in high and low mass channels, respectively; then $\mathrm{He}^{+}$and $\mathrm{H}^{+}$in high and low channels. The $\mathrm{He}^{+}$redundancy provides a measure of the relative response of the high and low mass channels. At this time, the low channel counting efficiency is reduced by channeltron gain fatigue, so that $\mathrm{H}^{+}$fluxes are higher by a factor of about 60 in comparison with $\mathrm{He}^{+}$(high mass channel) and $\mathrm{O}^{+}$than is suggested by the contouring.

Note that the event near 1935 UT separates regions of light ion polar wind flow, $\mathrm{H}^{+}$and $\mathrm{He}^{+}$being displaced significantly from the ram direction relative to their thermal widths, while $\mathrm{O}^{+}$is not. The event consists of a broadening of all three ion spin distributions, indicating heating of all species. Actual spin curves from before (upper panel), during (center panel) and after (lower panel) this event are shown in Figure 3. During the event the $\mathrm{O}^{+}$angular distribution is significantly broadened in the upgoing hemisphere but remains steep in the downgoing hemisphere, indicating a distribution function with a significant upward heat flux, i.e., an ion heat source below the observation altitude at about $5000 \mathrm{~km}$ for this event.

A hint of the nature of the heat source is visible in the $\mathrm{He}^{+}$angular distribution, which exhibits a slight local minimum in the vicinity of the magnetic field direction with a secondary maximum or knee at approximately $90^{\circ}$ spin phase angle. This is suggestive of conical structure and hence transverse acceleration below the spacecraft, though it is clear that the conic is asymmetric due to the ramming of the distribution. The conical structure might be more evident if the low energy ions were excluded by a retarding potential. Unfortunately, the radial head RPA is not operative during the period when the DE 1 orbit permits observation of such events. However, some energy distribution information is available for the $\mathrm{O}^{+}$ions. This is made possible by the relatively narrow energy response of the $\mathrm{O}^{+}$ channel (about $15 \mathrm{eV}$ ), which causes that channel to select more energetic $\mathrm{O}^{+}$ions with increasing RPA sweep, even though the RPA grid itself remains at spacecraft potential. Although precise energies cannot be assigned to the data at present, it is clear from these data that the more energetic tail of the $\mathrm{O}^{+}$distribution has conical lobes having a cone half angle of approximately $45^{\circ}$, indicating that the heating below the observation altitude is transverse in nature and occurs on the order of $1000-2000 \mathrm{~km}$ below the spacecraft, similar to the event described by M. Lockwood et al. (unpublished manuscript, 1984).

Figure 4 indicates schematically the nature of the distribution functions for $\mathrm{H}^{+}$and $\mathrm{O}^{+}$indicated by these observations. The figure shows velocity space in the frame 

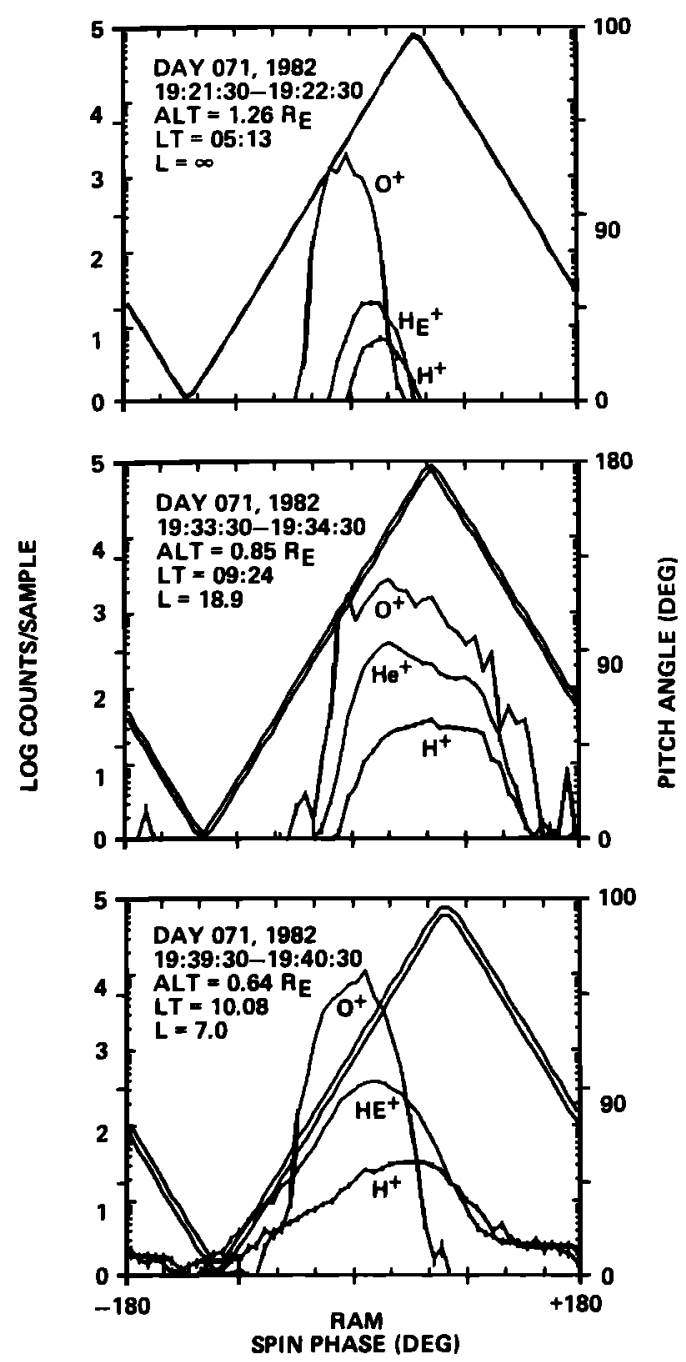

Fig. 3. Spin distribution curves for the major ion species during three subintervals of the data period shown in Plate 4.

of the spacecraft, with axes representing the direction of motion of the spacecraft $\left(V_{\mathrm{sc}}\right)$ and the direction perpendicular to $V_{\mathrm{sc}}$ and lying in the spin plane in the upgoing direction. The direction of the magnetic field is indicated by the line through the location of cold rammed plasma. In the upper panel appears a schematic representation of the polar wind distributions of $\mathrm{H}^{+}$and $\mathrm{O}^{+}$. The cold rammed $\mathrm{O}^{+}$appears as a peak centered at the antiram direction, while the $\mathrm{H}^{+}$is shown as an isotropic distribution moving upward along the magnetic field. The lower panel shows the type of distribution inferred from the observations of Figure 3. Note the magnetic field aligned asymmetry and the appearance of conic lobes at large speeds. These features constitute a significant heat and number flux of $\mathrm{O}^{+}$ions, indicating a heat source operating below the spacecraft and depositing heat preferentially in the perpendicular ion motions.

\section{DISCUSSION}

The four events described in the previous section are representative examples of the signatures of auroral acceleration processes as observed in the lowest energy topside ionospheric plasma. Three basic categories of low altitude signatures may be defined in terms of integral flux angular distributions. These are (1) conic (or transversely accelerated) signatures, as in the low altitude nightside event (Plate 2), (2) upwelling signatures, as in the low altitude dayside event (Figure 3), and (3) field aligned flow signatures, as seen in the polar wind light ion flows (Figure 3 ). At altitudes below $2 R_{E}$, the upwelling $\mathrm{O}^{+}$is the most common signature of auroral acceleration processes, with occurrence probabilities up to 0.8 in the prenoon auroral zone at 3000 to $5000 \mathrm{~km}$ altitude (M. Lockwood et al., published manuscript, 1984).

However, it should be noted that the actual distinctions between these categories are not sharp and that some overlap exists. The upwelling ion events exhibit conic structure in their energetic tails, suggesting that the distinction between the conic and upwelling categories is a matter of the degree to which transverse acceleration affects the thermal core of the ion distribution function. It is possible

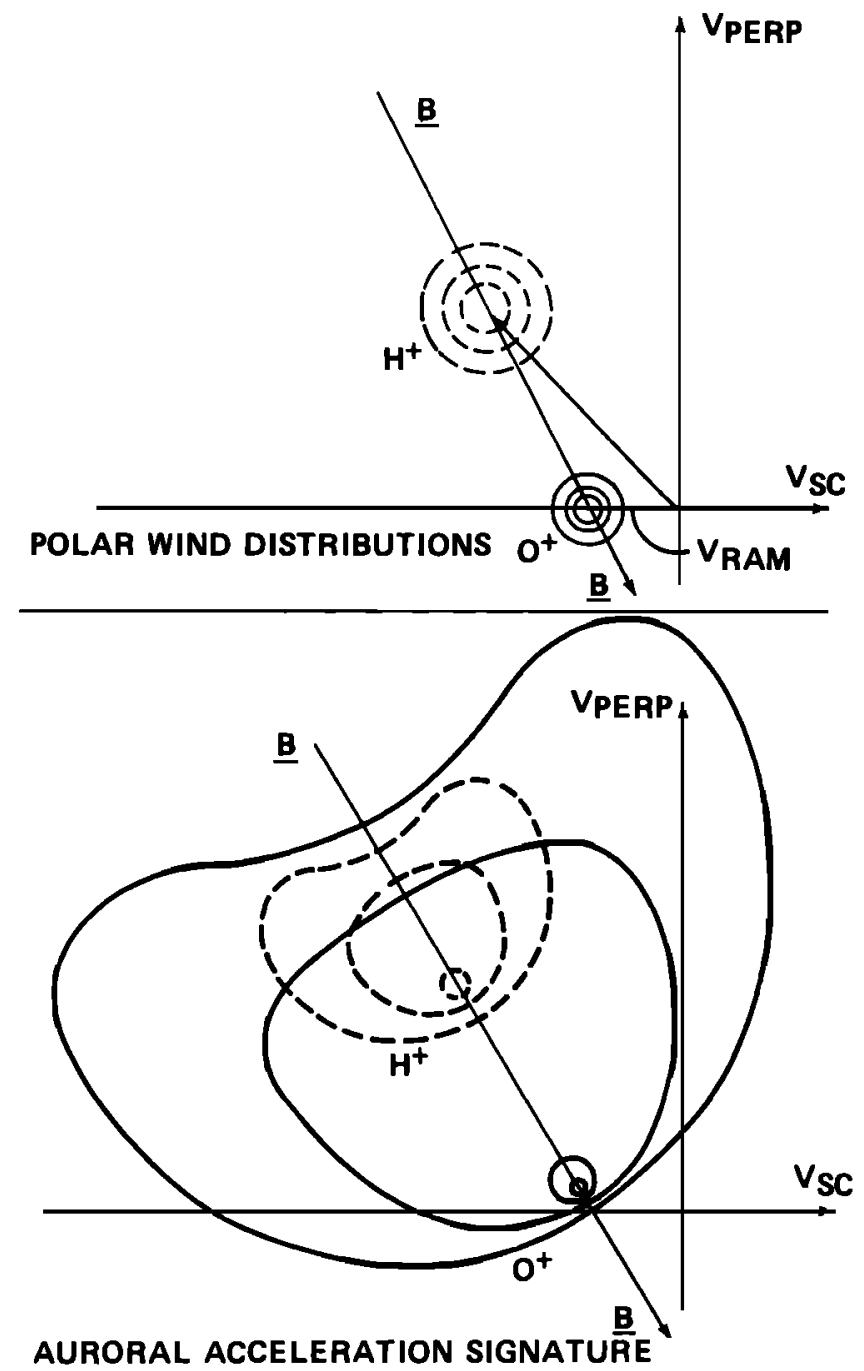

Fig. 4. Schematic illustration of the distribution functions inferred from the data of Plate 4 and Figure 3. The axes represent velocity space in the frame of the moving spacecraft, with axes aligned with the spacecraft velocity vector $\left(V_{\mathrm{sc}}\right)$ and with the upward normal to $V_{\mathrm{sc}}$. The magnetic field lies nearly in this plane, as indicated. The upper panel shows polar wind flow of $\mathrm{H}^{+}$relative to $\mathrm{O}^{+}$. The lower panel shows the signature of upward heat flux indicated by the data of Figure 3. 
to envision a continuum of behavior ranging from that seen in Plate 2, with the entire distribution appearing to be transversely accelerated, to that of Figure 3, in which a cold rammed population is retained and is in fact still dominant in the integral flux. At higher altitudes, one expects that the superthermal tails will dominate the integral flux, due to a combination of gravitational selection and the obscuring of the low energy core by increasingly positive spacecraft potential. Figure $1 c$ is an example at high altitude of comparable contributions from the rammed thermal core and from the superthermal tail. Earlier observations of low energy conics and transversely accelerated ions [Klumpar, 1979; Gorney et al., 1981] included only the superthermal tails of the distributions and were not mass selective, so that the above distinctions were not relevant.

It is probable that the high-altitude field aligned flow signature represents the evolution in altitude of the upwelling ion distribution. A comparison of Plates 3 and 4 suggests that the dispersive field aligned flows seen at high altitude originate in the dayside auroral zone upwelling ion events. The energy dispersion seen in the Plate 3 event requires that the flowing ions possess a broad distribution of parallel energies in the range zero to $50 \mathrm{eV}$, i.e., that they do not constitute a beam in the sense of having a field-aligned peak in velocity space. However, note that the energy dispersion in space can result in the appearance of a beam distribution at particular locations. It is possible that the distributions observed can be accounted for by low altitude transverse acceleration which extends to energies above that of gravitational binding, followed by adiabatic motions in the combined magnetospheric magnetic and electric fields. However, these observations reveal that these superthermal ionospheric outflows are inherently two-dimensional in nature, in contrast to most existing one-dimensional outflow models.

In contrast to these dayside signatures, nightside ion flows often exhibit beam distributions which are not attributable to dispersion of a localized source. The beam feature appears in RIMS data in the form of field aligned ion flux which is unretarded or retarded only for large values of the retarding potential. Beams are most apparent in the $\mathrm{O}^{+}$channel, as in Figure 1 , due to its differential response with bandwidth of about $15 \mathrm{eV}$. This suggests that parallel upward ion acceleration forming beams is primarily a nightside auroral phenomenon.

The observations shown here suggest relationships between the low and high altitude signatures of ion acceleration. The observation of transversely accelerated ions at low altitude in the nightside auroral zone and of " $X$ " angular distributions at higher altitudes suggests that parallel acceleration regions are superposed upon broader regions of transverse acceleration, and. this is confirmed by HAPI observations. However, there is reason to believe that transverse acceleration through resonant wave-particle interactions is associated with regions of downward rather than upward currents [Dusenbery and Lyons, 1981], and should accordingly be limited to regions of return current. An alternate mechanism for transverse acceleration invokes the presence of perpendicular electric fields having length scales comparable to the ion gyro-radius [Borovsky and Joyce, 1983; Greenspan, 1984]. The observation of conics in association with the edges of parallel acceleration structures as reported here may be a natural consequence of such a process. However, transverse acceleration extending throughout the region below a parallel acceleration region may require a completely new model for its description. The same might be said of the apparently toroidal $\mathrm{O}^{+}$distribution function occasionally observed.

\section{CONCLUSIONS}

We have presented representative examples of superthermal signatures of auroral ion acceleration processes. These observations complement earlier reports of energetic ion beams and conics, by simultaneously revealing the relationship between the thermal ionospheric plasma and superthermal features found in the energetic tail of each species distribution function. The superthermal features appear in sharp contrast to the signatures of light ion polar wind flow, often separating polar cap and subauroral regions of such flow. Polar wind flows of the light ions appear as shifts of light ion flux away from the ram direction and narrowing of the angular distribution consistent with a field aligned flowing distribution which is reasonably close to a drifting Maxwellian having transonic or supersonic drift speed. Such flows are invariably accompanied by $\mathrm{O}^{+}$flux distributions showing small shifts from ram, and little change in the angular distribution width, consistent with slow field aligned or convective flow of $\mathrm{O}^{+}$.

Low altitude superthermal effects generally include the development of an energetic tail with conical lobes in one or more ion species, indicating transverse acceleration at or below the spacecraft. In extreme cases, e.g., Plate 2, virtually all of the thermal plasma appears to have become transversely accelerated, to the point of forming a toroidal or ring distribution. In $\mathrm{O}^{+}$upwelling ion events, much of the upgoing $\mathrm{O}^{+}$flux is carried by the energetic tail which forms in the upgoing hemisphere and is unmatched in the downgoing hemisphere. In such cases the total ion distribution may be characterized as carrying a very large upward heat flux.

Comparison of high and low altitude superthermal ion signatures suggests that quite different processes are at work on the dayside and nightside auroral zones. On the dayside, a spatially confined source region combined with tailward convection over the polar cap leads to an inherently two-dimensional flow that disperses the ionospheric outflow in parallel velocity. The source distribution of parallel energies implied by the dispersion is very broad, extending from a few eV to greater than $50 \mathrm{eV}$ (the $>50 \mathrm{eV} \mathrm{H}^{+}$ions appear very close to the source region, while very low energy $\mathrm{O}^{+}$ions are convected well into the polar cap). The field aligned outflow seen at high altitude can have the appearance of a beam, but this appears to be due to the parallel velocity dispersion rather than to preferential acceleration to a particular parallel speed or energy. We interpret the energy distribution of this polar cap flow as the result of transverse acceleration followed by adiabatic "folding" of the resulting conics in the diverging magnetic field, with lateral convection. It is very interesting to note that the clear mass dispersion observed implies an energization mechanism which imparts similar energy distributions, rather than similar velocity distributions, to each species. This suggests an electrostatic mechanism, but a simple parallel potential drop is incon- 
sistent with the broad range of energies implied by the observations. Proper deconvolution of the source energy distribution will require a detailed model of this flow region, including the effects of gravity, a self-consistent electric field, and the presence of light ion polar wind at very low energies.

On the nightside, observation of the $\mathrm{X}$ signature formed by ion conics, with appearance of beams at the vertex of the $X$, suggests that transverse acceleration is associated with the edges of the auroral parallel acceleration region. Though information about transverse acceleration tends to be obscured when parallel acceleration appears in the center of these events, HAPI ion data indicate that transverse acceleration is occurring there as well. It is clear that the transverse acceleration region moves down in altitude with close proximity to the parallel acceleration region. Morover, this descent in altitude is associated with increased $\mathrm{O}^{+}$content in the event of Plate 1 and Figure 1. This raises the interesting question of ion acceleration at the interface of the parallel acceleration region and the topside ionosphere.

Much more work is needed to relate these observations to the larger picture of ionospheric outflow in auroral contexts. In particular, comparisons with other data sets involving field aligned currents, plasma and neutral convection, electric and magnetic fields and waves, and more energetic particles may be expected to help answer the numerous open questions regarding the mechanism of transverse acceleration and its interaction with the ionospheric plasma.

Acknowledgments. The authors are indebted to the engineering and science staff of the University of Texas at Dallas and to the RIMS team at Marshall Space Flight Center. We are grateful to the programming staff of the Intergraph and Boeing Corporations for assistance with the data reduction software and to the MSFC/SCAN network for support of the graphics analysis. Significant data analysis contributions were also made by Barbara Giles. We are indebted to M. Sugiura for providing magnetometer data for spacecraft aspect. Support for M. Lockwood was provided by the National Research Council, under their Resident Research Associateship program.

The Editor thanks J. F. Fennell and another referee for their assistance in evaluating this paper.

\section{REFERENCES}

Borovsky, J. E., and G. Joyce, Numerically simulated two-dimensional auroral double layers, J. Geophys. Res., 88, 3116, 1983.
Burch, J. L., P. H. Reiff, R. A. Heelis, J. D. Winningham, W. B. Hanson, C. Gurgiolo, J. D. Menietti, R. A. Hoffman, and J. N Barfield, Plasma injection and transport in the mid altitude polar cusp, Geophys. Res. Lett., 9, 921, 1982.

Chappell, C. R., S. A. Fields, C. R. Baugher, J. H. Hoffman, W. B. Hanson, W. W. Wright, and H. D. Hammack, The retarding ion mass spectrometer on Dynamics Explorer-A, Space Sci. Instrum., $5,477,1982$.

Chiu, Y. T., J. M. Cornwall, J. F. Fennel, D. J. Gorney, and P. F. Mizera, Auroral plasmas in the evening sector: Satellite observations and theoretical interpretations, Space Sci. Rev., 35, 211, 1983.

Dusenbery, P. B., and L. R. Lyons, Generation of ion conic distributions by upgoing ionospheric electrons, $J$. Geophys. Res., $86,7627,1981$.

Gorney, D. J., A. Clarke, D. Croley, J. Fennel, J. Luhmann, and P. Mizera, The distribution of ion beams and conics below 8000 km altitude, J. Geophys. Res., 86, 83, 1981.

Greenspan, M. E., Effects of oblique double layers on upgoing ion pitch angle and gyrophase, J. Geophys. Res., 89, 2842, 1984.

Horwitz, J. L., The ionosphere as a source of magnetospheric ions, Rev. Geophys. Space Phys., 20, 929, 1982.

Johnson, R. G., Energetic ion composition in the Earth's magnetosphere, Rev. Geophys. Space Phys., 17, 696, 1979.

Klumpar, D. M., Transversely accelerated ions: An ionospheric source of hot magnetospheric ions, J. Geophys. Res., 84, 4229, 1979.

Lockwood, M., and J. E. Titheridge, Departures from diffusive equilibrium in the topside $F$-layer from satellite soundings, J. Atmos. Terr. Phys., 44, 425, 1982.

Moore, T. E., Superthermal ionospheric outflows, Rev. Geophys. Space Phys., 22, 264, 1984.

Nagai, T., J. H. Waite, Jr., J. L. Green, C. R. Chappell, R. C. Olsen, and R. H. Comfort, First measurements of supersonic polar wind in the polar magnetosphere, Geophys. Res. Lett., 11, $669,1984$.

Reiff, P. H., T. W. Hill, and J. L. Burch, Solar wind plasma injection at the dayside magnetospheric cusp, J. Geophys. Res., 82, $479,1977$.

Waite, J. H., Jr., T. Nagai, J. F. E. Johnson, C. R. Chappell, J. L. Burch, T. L. Killeen, P. B. Hays, G. R. Carignan, W. K. Peterson, and E. G. Shelley, Escape of suprathermal $\mathrm{O}^{+}$ions in the polar cap, J. Geophys. Res., in press, 1985.

C. R. Chappell, M. Lockwood, T. E. Moore, and J. H. Waite, Jr., Code ES53, Space Science Laboratory, NASA Marshall Space Flight Center, Huntsville, AL 35812.

(Received July 23, 1984;

revised October 3, 1984;

accepted October 4, 1984.) 
DE-1 RIMS 20 OCT 1981 (DAY 293)

a) RADIAL HEAD: H+: LOW MASS CHANNEL
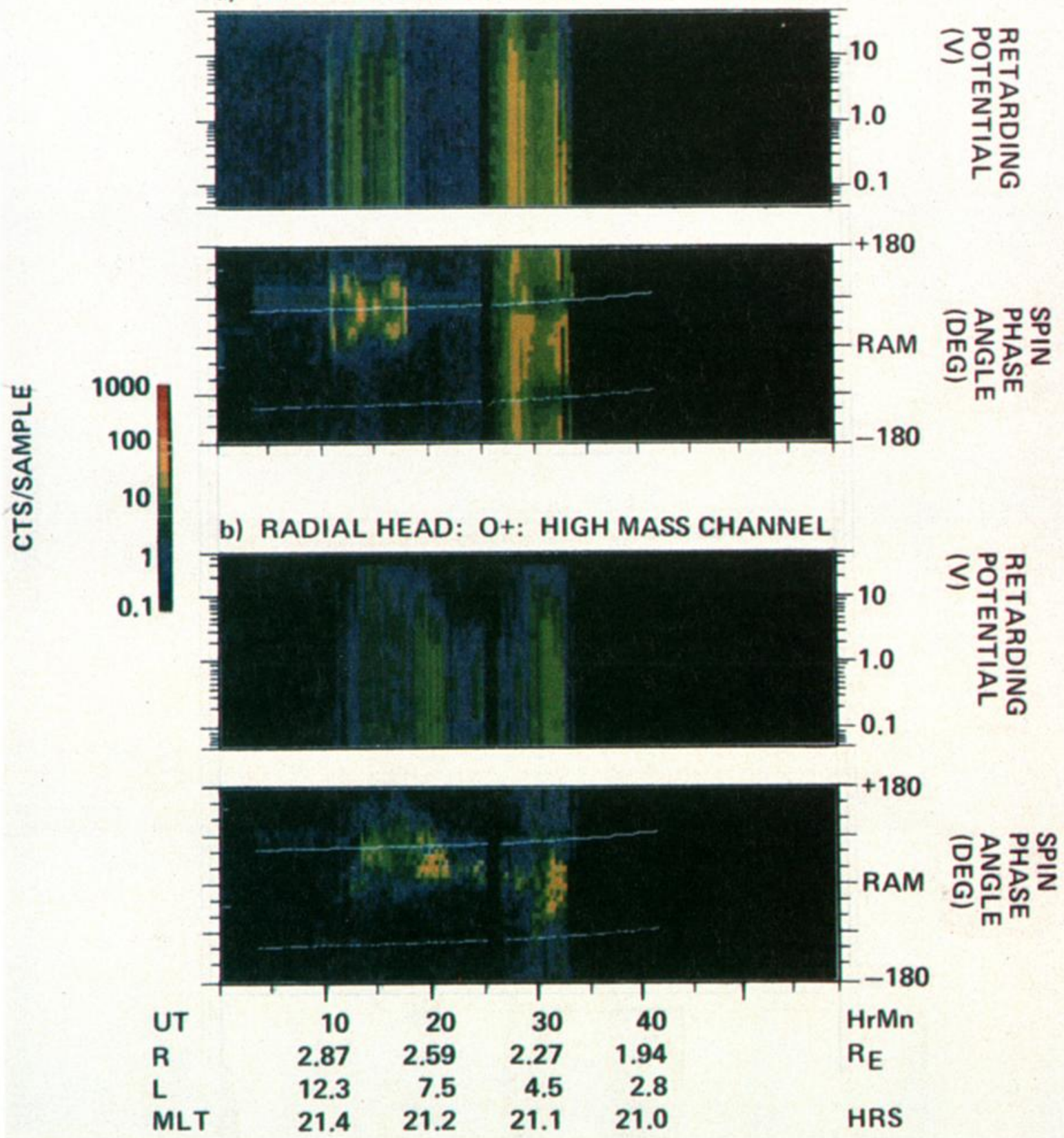

Plate 1 [Moore et al.]. DE 1 RIMS retarding potential and spin spectrograms for $\mathrm{H}^{+}$(top) and $\mathrm{O}^{+}$(bottom) during a high altitude nightside pass over the auroral zone. The quantity contoured is the detector count rate, a measure of the integral flux above an energy corresponding ot the RPA potential in the case of the RPA spectrograms, or the integral flux above spacecraft potential in the case of the spin spectrograms. 
DE RIMS 20 APRIL 1982 (DAY 110)

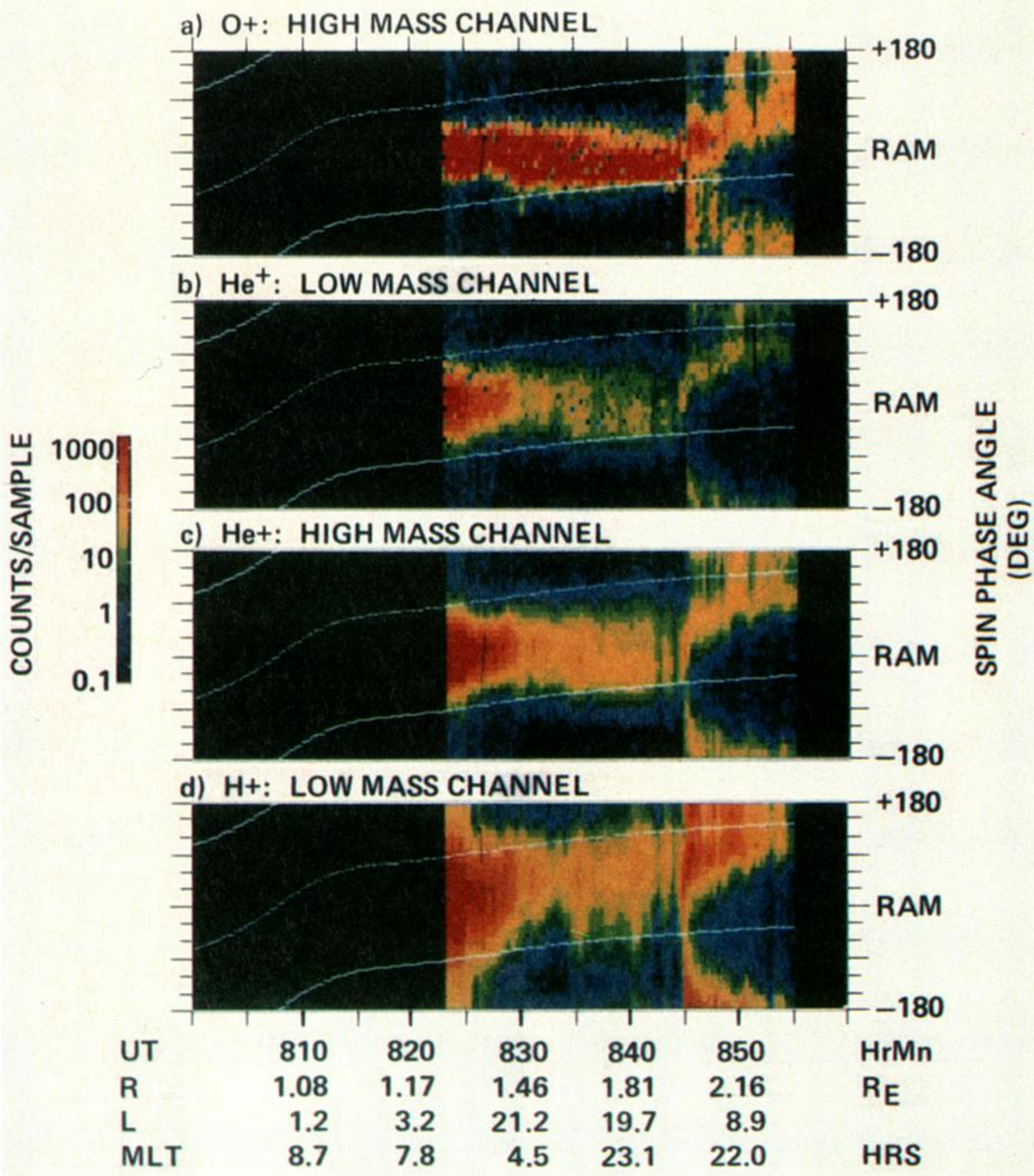

Plate 2 [Moore et al.]. DE 1 RIMS spin spectrograms for $\mathrm{O}^{+}$(top), $\mathrm{He}^{+}$low mass channel (top center), $\mathrm{He}^{+}$high mass channel (bottom center), and $\mathrm{H}^{+}$(bottom) during a low altitude nightside pass over the auroral zone. 


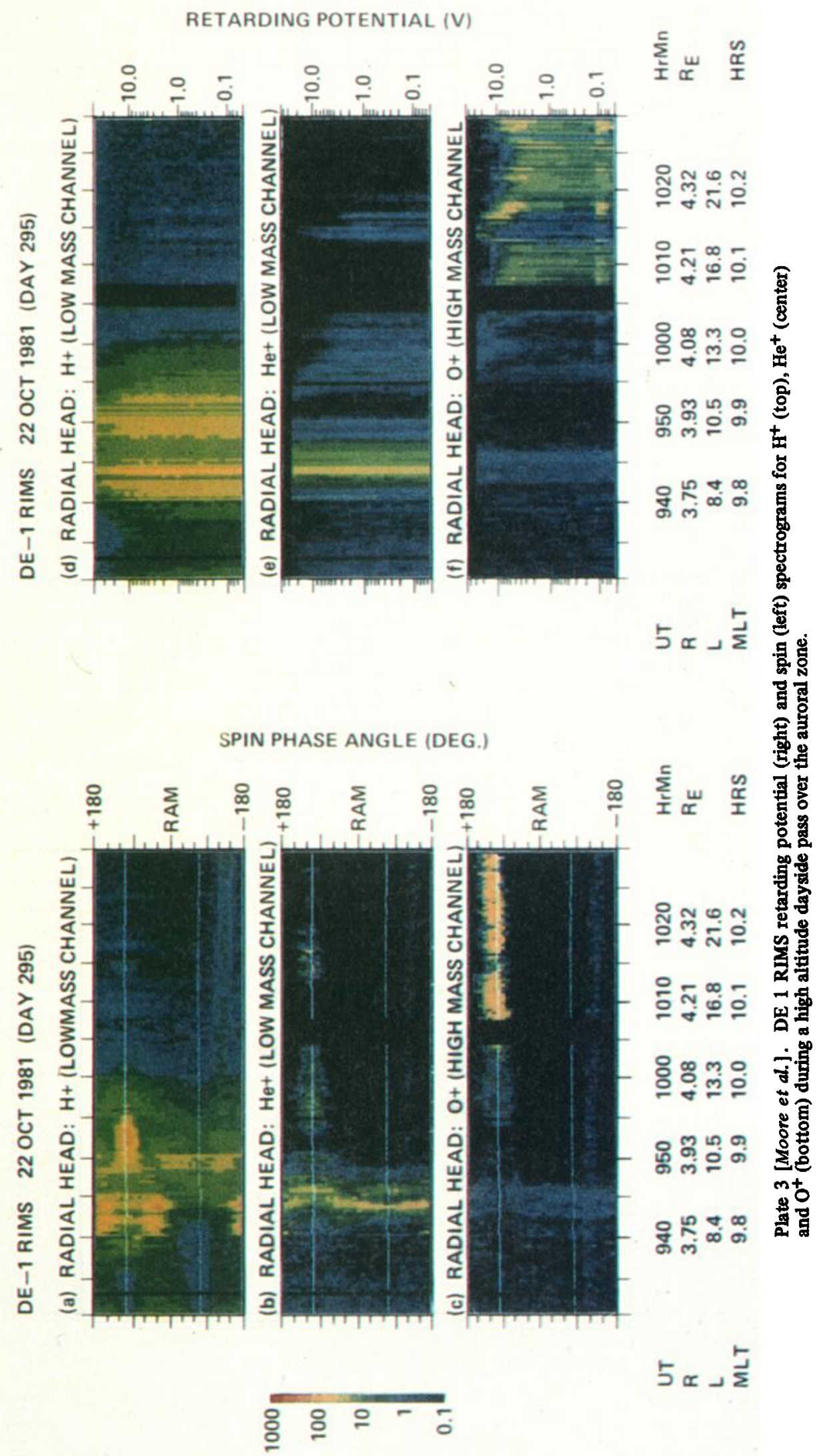




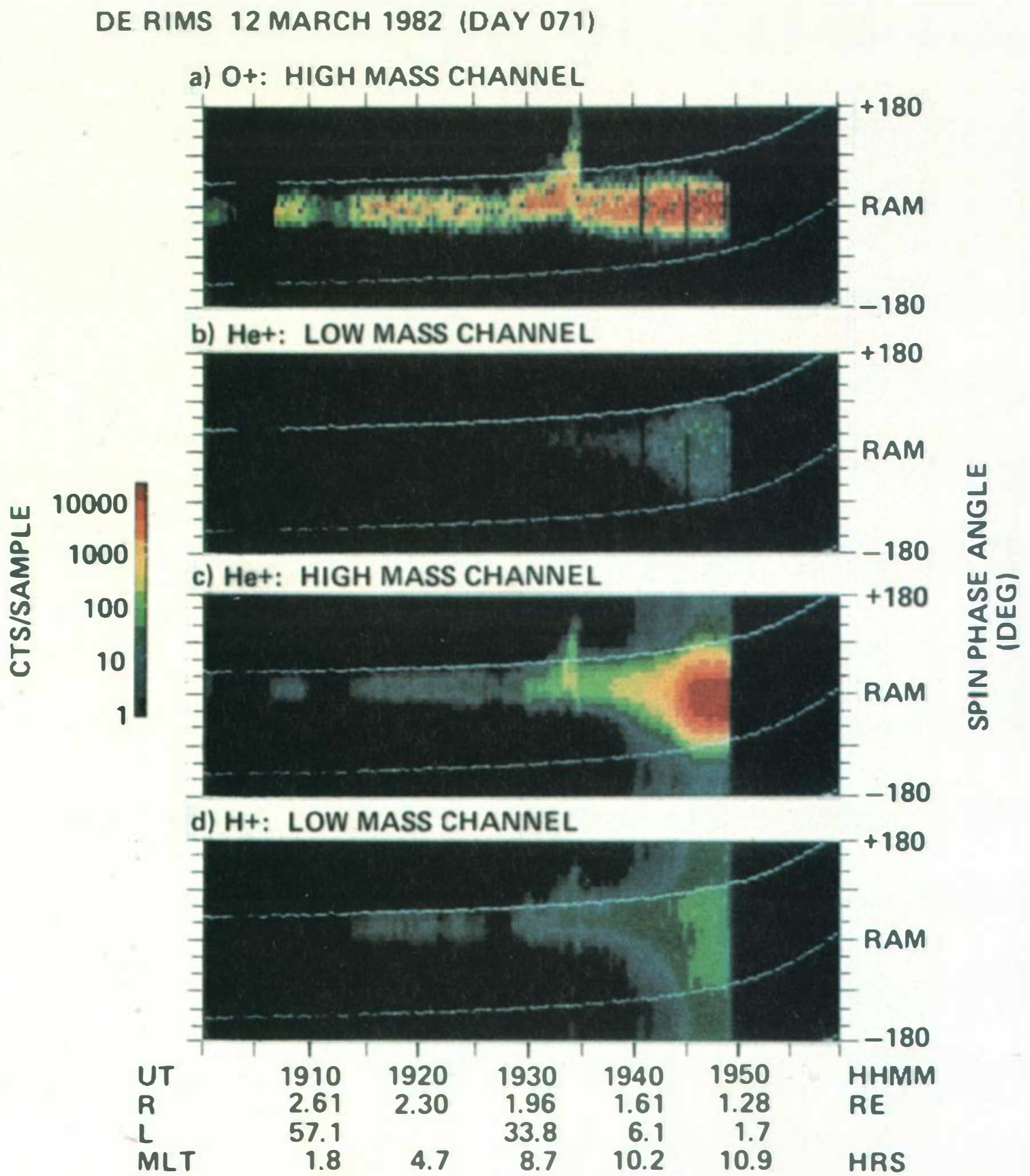

Rave 4 [Moore et d.]. DE 1 RIMS spin spectrograms for $\mathrm{O}^{+}$(top), $\mathrm{He}^{+}$low mass channel (top center), $\mathrm{He}^{+}$high mass artiom center), and $\mathrm{H}^{+}$(bottom) during a low altitude dayside pass over the auroral zone. 